\title{
Tylosis in a Patient With Howel-Evans Syndrome: Management With Acitretin
}

\author{
Sabra M. Abner, MD; Audra Isaac, MD; Courtney R. Schadt, MD
}

\section{PRACTICE POINTS}

- Keratoderma can be especially painful for patients and can have a great impact on their quality of life. For these patients, acitretin should be considered when topical therapies have failed.

- Howel-Evans syndrome is an autosomal-dominant condition that predominantly presents with plantar keratoderma and has a high risk for esophageal cancer.

To the Editor:

Tylosis with esophageal cancer was first described by Howel-Evans et $\mathrm{al}^{1}$ in 1958 in a family from Liverpool, England. The disease is inherited in an autosomaldominant fashion with a mutation in the tylosis with esophageal cancer gene, TOC. ${ }^{2}$ The keratoderma associated with this syndrome has been reported to be focal in nature, painful, and primarily involving the plantar surfaces. ${ }^{3}$ Palmar involvement has been reported to manifest as calluses in patients who use their hands for manual labor. ${ }^{4}$ Oral leukoplakia also has been described in this syndrome; ${ }^{5}$ however, long-term follow-up in one family demonstrated a benign course. ${ }^{6}$ Herein, we describe a case of painful tylosis in a patient with Howel-Evans syndrome who was successfully treated with acitretin.

A 50-year-old man presented to clinic for evaluation of hyperkeratosis of the palms and soles that began when he was a teenager. He reported the soles of the feet often were painful, especially without shoes (Figure, A). He used many over-the-counter emollients and tried both prescription and nonprescription keratolytics. At presentation, he was mechanically paring down some of the thickness of the calluses to decrease the pain.

There was no relevant medical history, he had no history of smoking, he consumed more than 1 alcoholic drink per day, and he denied illicit drug use. The patient was not on any other medications. His family history revealed that his father also had the same hyperkeratosis of the palms and soles and died from esophageal carcinoma at an early age. It was determined that his father had tylosis with esophageal carcinoma (Howel-Evans syndrome). (The patient's pedigree previously was published. ${ }^{3,4}$ ) Physical examination at presentation revealed plantar hyperkeratosis limited mainly to areas of pressure. His hands had mild hyperkeratosis on the distal fingers. No mucosa leukoplakia was identified.

Treatment options were discussed, and because the pain associated with the plantar keratoderma was interfering with his quality of life (QOL), acitretin was started. The initial dosage was $10 \mathrm{mg}$ daily for 2 weeks and subsequently was increased to $25 \mathrm{mg}$ daily. He has been maintained on this dosage for more than a year. An attempt was made to increase acitretin to $50 \mathrm{mg}$ daily; however, he could not tolerate the dryness and peeling of the hands caused by the higher dosage. A fasting lipid panel and hepatic function panel performed every 3 months was within reference range. He had a remarkable decrease in the hyperkeratosis 2 months after starting therapy (Figure, B) and most importantly a decrease

Drs. Abner and Schadt are from the Division of Dermatology, University of Louisville, Kentucky. Dr. Isaac is from the Division of Dermatology, Eastern Carolina University, Greenville, North Carolina.

The authors report no conflict of interest.

Correspondence: Sabra M. Abner, MD, University of Louisville, Division of Dermatology, 3810 Springhurst Blvd, Ste 200 , Louisville, KY 40241

(sabraabner@gmail.com).

doi:10.12788/cutis.0114 
A, Thick, yellow, hyperkeratotic plaques on the left plantar surface prior to treatment with acitretin. B, Hyperkeratosis diminished after 7 months of acitretin therapy.
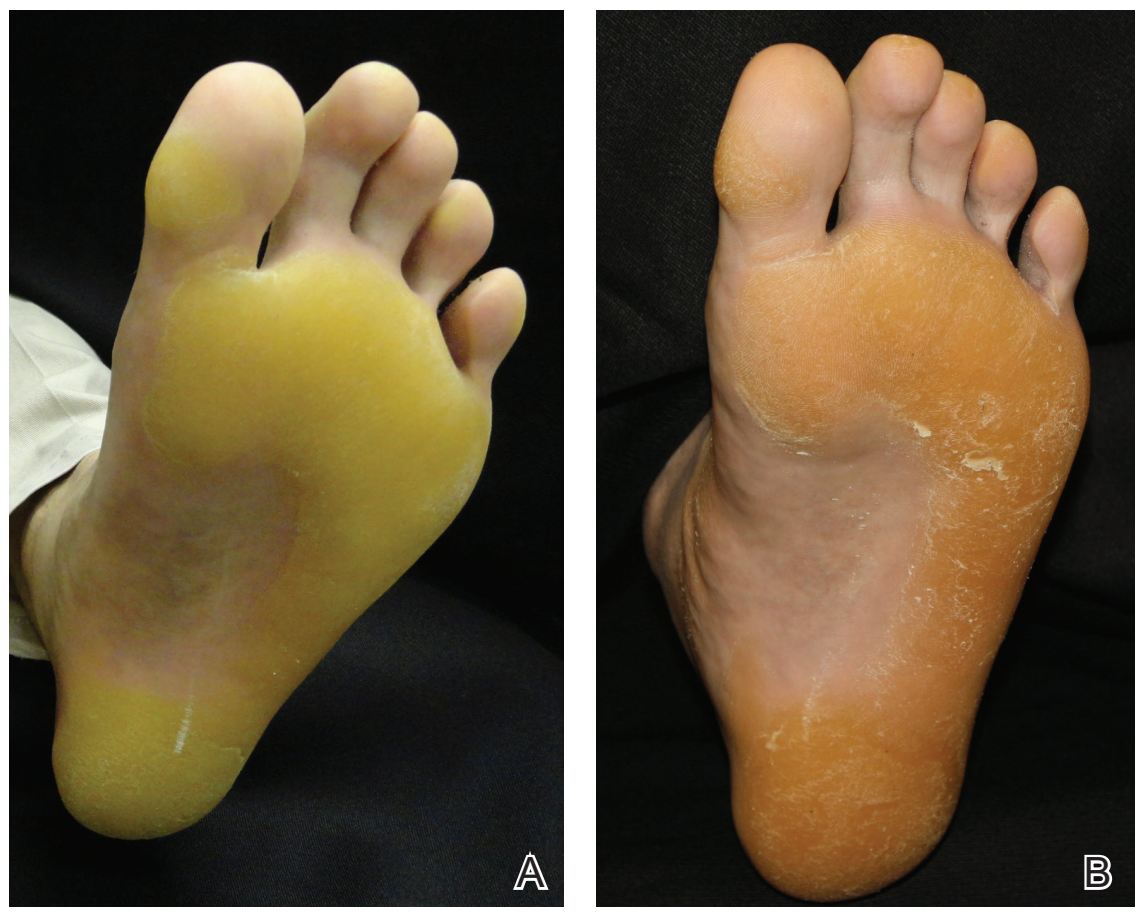

in pain associated with it. His QOL notably improved, enabling him to participate in sporting events with his children without severe pain. This patient was referred to gastroenterology where an esophagogastroduodenoscopy was performed and no concerning lesions were found. He was continued on this dose for 2 years. He moved to a new town, and our most recent update from him was that he was taking acitretin intermittently before big sporting events with his children.

The use of systemic retinoids has long been known to be effective in the treatment of disorders of keratinization. Recommended monitoring guidelines include a baseline complete blood cell count, renal function, hepatic function, and fasting lipid panel, which should be repeated every 3 months focusing on the hepatic function and lipid panel, as retinoids rarely cause hematologic or renal abnormalities. ${ }^{7}$ Our patient's baseline laboratory test results were within reference range, and we repeated a fasting lipid and hepatic function panel every 3 months without any abnormalities.

Diffuse idiopathic skeletal hyperostosis (DISH), the ossification of ligaments and entheses often of the spine, is a potential complication of long-term use of oral retinoids. There are no consensus guidelines on screening for this complication, but baseline and annual radiographs seem reasonable. A 1996 study concluded that if DISH occurs, it is likely to be sporadic in a predisposed patient, as their data did not find any statistically significant relationship between the treatment or the cumulative dose and the prevalence and severity of DISH, degenerative changes, and osteoporosis. ${ }^{8}$ When annual screening is declined, imaging could be performed if a new skeletal concern were to arise in patients on long-term therapy. ${ }^{7}$
We discussed the skeletal concerns with our patient and he declined baseline or annual radiographs, but we will follow him with a rheumatologic review of systems. We feel this approach is reasonable, as our patient is a healthy adult in his 50s with no prior retinoid exposure and is on a low to moderate dose.

We report a case of Howel-Evans keratoderma successfully managed with acitretin. In patients with painful keratoderma that is interfering with QOL, low-dose acitretin can be used to diminish these symptoms.

\section{REFERENCES}

1. Howel-Evans W, McConnell RB, Clarke CA, et al. Carcinoma of the oesophagus with keratosis palmaris et plantaris (tylosis): a study of two families. Q J Med. 1958;27:413-429.

2. Rogaev EI, Rogaeva EA, Ginter EK, et al. Identification of the genetic locus for keratosis palmaris et plantaris on chromosome 17 near the RARA and keratin type I genes. Nat Genet. 1993;5:158-162.

3. Stevens HP, Kelsell DP, Bryant SP, et al. Linkage of an American pedigree with palmoplantar keratoderma and malignancy (palmoplantar ectodermal dysplasia type III) to 17q24. literature survey and proposed updated classification of the keratodermas. Arch Dermatol. 1996;132:640-651.

4. Marger RS, Marger D. Carcinoma of the esophagus and tylosis. a lethal genetic combination. Cancer. 1993;72:17-19.

5. Tyldesley WR. Oral leukoplakia associated with tylosis and esophageal carcinoma. J Oral Pathol. 1974;3:62-70.

6. Ellis A, Field JK, Field EA, et al. Tylosis associated with carcinoma of the oesophagus and oral leukoplakia in a large Liverpool family-a review of six generations. Eur J Cancer B Oral Oncol. 1994;30B:102-112.

7. Wu J, Wolverton S. Systemic retinoids. In: Wolverton S, ed. Comprehensive Dermatologic Drug Therapy. 4th ed. Edinburgh, Scotland: Elsevier; 2020:245-262.

8. Van Dooren-Greebe RJ, Lemmens JA, De Boo T, et al. Prolonged treatment with oral retinoids in adults: no influence on the frequency and severity of spinal abnormalities. Br J Dermatol. 1996;134:71-76. 